ORIGINAL ARTICLE

\title{
Node retrieval in axillary lymph node dissections: recommendations for minimum numbers to be confident about node negative status
}

\author{
J E A Somner, J M J Dixon, J S J Thomas
}

J Clin Pathol 2004;57:845-848. doi: 10.1136/icp.2003.015560

See end of article for authors' affiliations

.....................

Correspondence to:

Dr J Thomas, Department

of Pathology, Western

General Hospital,

Edinburgh EH4 2XU, UK;

jeremy.thomas2@

btinternet.com

Accepted for publication 10 February 2004

\begin{abstract}
Aims: To determine the minimum number of lymph nodes needed in an axillary lymph node dissection (ALND) specimen to be confident that the axilla is free from metastases.

Methods: The Edinburgh Breast Unit selects patients with large and high grade tumours for ALND; 609 consecutive ALNDs performed between October 1999 and December 2002 were reviewed. Full data about the underlying invasive breast cancer were available for 520 patients. Data were collected regarding number of positive nodes and total number of nodes collected, tumour size and grade, and presence of lymphovascular invasion.

Results: Axillary node metastases were seen in $64 \%$ of patients. The mean number of positive nodes found was 3.56 , with a mean of 17.9 nodes collected. The highest proportion of patients with lymph node metastases were in the group with 16-20 nodes recovered/specimen (68\%); specimens with $>20$ nodes recovered did not have a higher rate of nodal involvement. There was a significant difference between the proportion of metastasis positive specimens in those with 1-15 nodes recovered (58.5\%) and those with 16 or more recovered (69.1\%). A linear association test showed a direct correlation between the number of nodes collected and presence of node metastasis $(p=0.0005)$.

Conclusions: Although there is no minimum number of nodes that should be recovered in an ALND specimen, 16 nodes should be regarded as a target to ensure a high level of confidence that the nodes are negative. Node positivity in an ALND specimen appears to obey the law of diminishing returns.
\end{abstract}

A xillary lymph node dissection (ALND) provides important prognostic information that influences crucial management decisions. Historically, its role has been in staging and planning adjuvant chemotherapy, and also in local control of cancer in both node positive and node negative tumours. ${ }^{1}$ Node positive status and the number of nodes containing metastases is associated with an increased risk of local recurrence and disease progression. ${ }^{2-5}$ A cancer with four or more positive nodes has a particularly aggressive phenotype. ${ }^{6}$ Node status is also considered to be a surrogate marker for the number of acquired and expressed genetic alterations. ${ }^{7}$ Unfortunately, ALND carries a $60 \%$ risk of significant morbidity including lymphoedema, sensory numbness, and limitation of arm motion and strength. ${ }^{8}$ In view of these complications, the Edinburgh Breast Unit has a policy of selecting patients with cancers that have a poorer prognosis for ALND and managing the axilla of patients with a potentially better prognosis by axillary node sampling and/ or sentinel node biopsy.

\begin{abstract}
"Although some centres set a desirable minimum for the numbers of nodes retrieved in an axillary lymph node dissection specimen the evidence base for the said numbers is limited and has prompted our study"
\end{abstract}

In light of the known complications and changing trends in the presentation of breast cancer, the role of ALND is under debate. Sentinel lymph node detection is showing promise and is being used in clinically node negative patients to avoid indiscriminate application of ALND. In spite of progress towards less invasive techniques and earlier detection of cancers, $5-10 \%$ of tumours $<0.5 \mathrm{~cm}$ in diameter and $10-20 \%$ of tumours $0.6-1 \mathrm{~cm}$ in diameter will be lymph node positive, and $15-30 \%$ of clinically node negative patients will develop axillary failure if the axilla is left untreated by either radiotherapy or dissection." Management of the axilla by ALND is necessary in patients for whom axillary radiotherapy is unsuitable. Patients with clinically palpable axillary nodes and those with positive sentinel nodes are also advised to undergo ALND. Current guidelines suggest that at least 10 nodes should be examined for optimal prognostic staging. ${ }^{10} 11$ Although some centres set a desirable minimum for the numbers of nodes retrieved in an ALND specimen the evidence base for the said numbers is limited and has prompted our study. Studies in patients with colorectal cancer have indicated that it is vital to assess enough lymph nodes to be sure of accurate disease staging. ${ }^{12-15}$ Our study reviews a large series of patients undergoing ALND in a specialised breast unit and aims to establish recommendations for the number of lymph nodes to be recovered from each ALND specimen, thereby helping to set quality standards for future practice.

\section{MATERIALS AND METHODS}

The pathology reports of 609 consecutive patients undergoing ALND between October 1999 and December 2002 in the Edinburgh Breast Unit were audited. Data were collected for the number of positive nodes and total number of nodes collected, tumour size and grade, and the presence of lymphovascular invasion. Cases were only included if the patient had confirmed invasive breast carcinoma and if a complete level III (removal of all nodes from levels I, II, and III) ALND was carried out. ALND specimens are not routinely labelled by the surgeon to identify separately levels I, II, and III.

Abbreviations: ALND, axillary lymph node dissection 
Cases with a known history of distant metastasis were excluded. The linear by linear association test was used for statistical analysis (statXact User Manual; CYTEL Software, Cambridge, Massachusetts, USA). Other statistical tests included Pearson's $\chi^{2}$ test and the Student's $t$ test (SPSS Inc, Chicago, Illinois, USA).

\section{RESULTS}

\section{Lymph node metastases}

Of the 609 ALNDs analysed, in 89 cases (1) there was limited information about the primary tumour; (2) the patients had extensive ductal carcinoma in situ and focal invasion that was ungradeable; or (3) the patients had previously received chemotherapy. Fifty nine of these 89 excluded patients had node metastases. No further analysis was carried out on this group. Five hundred and twenty patients had complete information about the primary tumour. These patients formed the definitive study group. Sixty four per cent of these patients had axillary node metastases. The mean number of positive nodes found was 3.56 (median, 1; range, 0-35), with a mean of 17.9 nodes collected for each specimen (median, 17; range, 2-47). Eight per cent of patients had 10 or fewer lymph nodes collected, $28 \%$ had 11 to 15 nodes, and $64 \%$ had $>16$ nodes. The case with only two nodes identified was in fact an extremely large tumour mass of matted nodes and striated muscle within the axilla, inside which two discrete nodes could be identified. The relation between the number of lymph nodes recovered for each specimen and the proportion of specimens with detectable metastases was examined with a test for linear by linear association (fig l). The proportion of specimens with metastases was strongly and positively related to the number of lymph nodes recovered $(\mathrm{p}=0.0005)$.

The highest proportion of patients with lymph node metastases occurred in the group with 16-20 nodes recovered for each specimen (68\%); specimens with $>20$ nodes recovered did not have a higher proportion (fig 1). There was a significant difference in the proportion of metastasis positive specimens between those who had $1-15$ nodes recovered $(58.5 \%)$ and those who had 16 or more recovered (69.1\%; $\mathrm{p}=0.006)$.

Cases were also categorised according to the number of positive nodes identified from 0 to $>5$. There was no

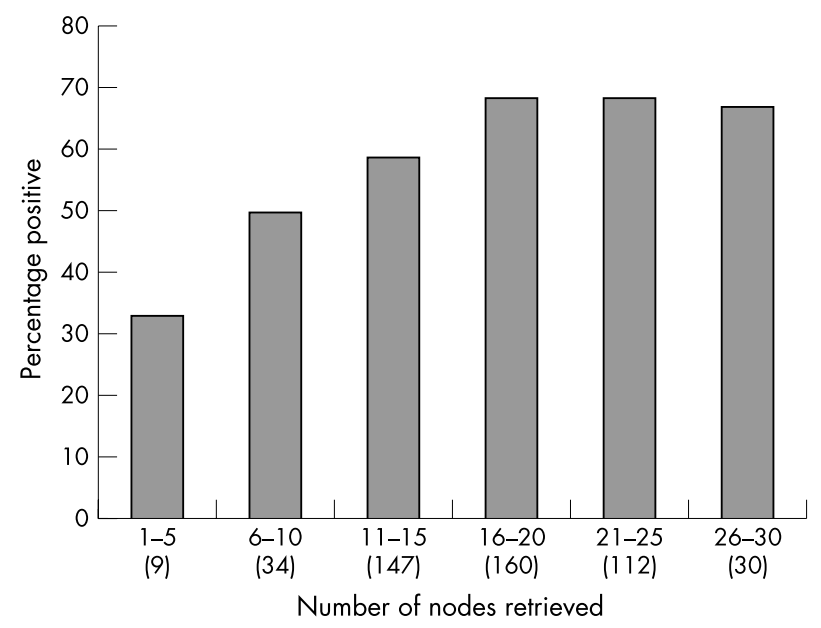

Figure 1 Percentage of cases with positive nodes compared with the number of nodes retrieved in each case. Results are grouped as follows: 1-5 nodes, 6-10 nodes, 11-15 nodes, 16-20 nodes, 21-25 nodes, and $26-30$ nodes, with the total number of cases for each group in parentheses.
Table 1 The relation between tumour grade and nodal status

\begin{tabular}{lllllll}
\hline Grade & Negative & $\%$ & Positive & $\%$ & Total & $\%$ \\
\hline 1 & 25 & 60 & 17 & 40 & 42 & 8 \\
2 & 71 & 32 & 150 & 68 & 221 & 43 \\
3 & 90 & 35 & 167 & 65 & 257 & 49 \\
Total & 186 & 36 & 334 & 64 & 520 & \\
\hline
\end{tabular}

significant difference in the number of nodes retrieved for each case for each category.

Lymphatic/vascular invasion correlated positively with node metastases, both in terms of whether or not nodes were involved and in terms of the number of nodes involved (fig 2).

\section{Influence of tumour grade}

Five hundred and twenty reports $(85 \%$ of the total original study population) contained information about tumour grade. These were analysed for the effect of grade on node status. Eight per cent of these cases were grade 1, 43\% were grade 2 , and $49 \%$ were grade 3 (table 1 ).

Grade 1 tumours had significantly fewer positive nodes than grade $2(p=0.002)$ and grade $3(p=0.006)$ tumours. There was no significant difference between grade 2 and 3 tumours. There was no significant difference between the number of nodes gathered for tumours of each grade (means: 16,18 , and 18 , respectively).

\section{Analysis of the tumours on the basis of node positivity and tumour size}

Thirty six per cent of the whole population was node negative (TNM N0), 34\% had between 1 and 3 positive nodes, and 30\% had four or more positive nodes. There was no significant difference between the tumour size of those specimens with no lymph node metastases and those with one to three lymph node metastases. However, tumours in both these groups were considerably smaller than in the group with four or more positive lymph nodes $(\mathrm{p}=0.002)$ (fig 3 ).

\section{DISCUSSION}

The Edinburgh Breast Unit has a selective policy for the use of ALND. The criteria for selection are not absolute but are as follows:

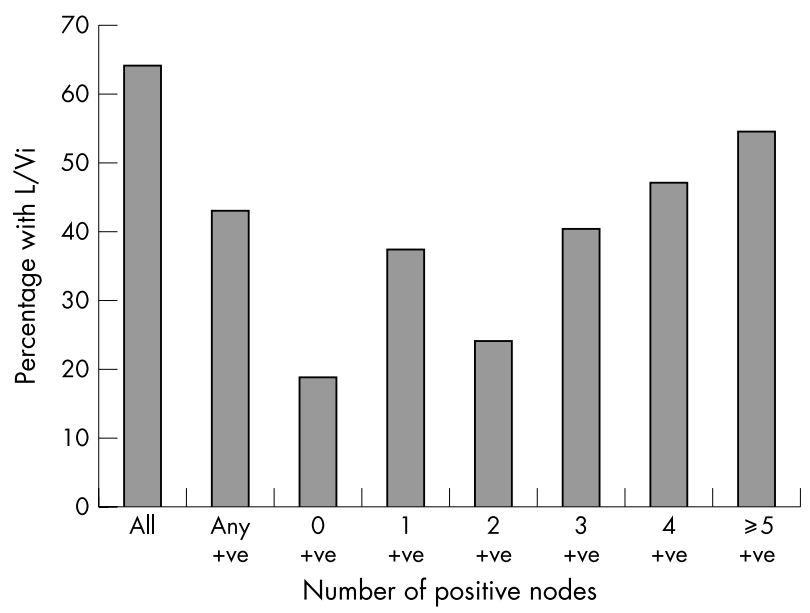

Figure 2 Percentage of patients with lymphatic invasion (L/Vi) when divided into groups on the basis of number of positive nodes. 




Figure 3 Mean tumour size compared with node status (negative, 1-3 or $>4$ positive).

- Clinical, mammographic, or cytological evidence of involved axillary nodes.

- If at the time of mastectomy, axillary clearance would avoid the need for postoperative radiotherapy, which would be otherwise contraindicated for medical or radiotherapeutic reasons, or for reasons of patient preference.

- Consider for younger women for whom the systemic treatment would be changed in the light of extensive nodal involvement.

- Patients with tumours $>2 \mathrm{~cm}$.

- Patients with grade 3 tumours on core biopsy.

- Evidence of vascular or lymphatic invasion on core biopsy.

- Patient preference.

This explains the relatively high rate of node positivity in our study in comparison with an overall node positive rate for the unit as a whole, which is in the order of $30 \%$ and is comparable with overall figures for other large breast units. For those patients with lower risk primary tumours, the axilla is managed by either node sampling and/or sentinel node biopsy. Previous studies have shown comparability between node sampling/sentinel node biopsy and ALND in terms of detection of axillary metastases, ${ }^{16}{ }^{17}$ but ALND has a therapeutic role and is more appropriate for those patients with a higher risk of metastatic disease. Our results also indicate that our selection process for ALND is effective, although it could be argued that those patients with node negative clearances had been overtreated.

Previous studies have indicated that in women with pathologically node negative invasive breast cancer in the absence of systemic treatment the number of nodes collected has an important and significant effect on local relapse and overall survival. ${ }^{18}$ Node negative women who had $<14$ lymph nodes collected had a 10 year survival $10 \%$ less than those with $>14$ lymph nodes collected. ${ }^{19}$ This pattern was also seen in women who had less than 10 nodes and less than 15 nodes collected. ${ }^{20}$

The number of nodes collected and analysed depends not only on the diligence of the pathologist but also on the extent of axillary dissection. ${ }^{21}$ It may also be influenced by neoadjuvant chemotherapy, which can make lymph nodes

\section{Take home messages}

- In patients undergoing axillary lymph node dissection (ANLD), the proportion of node positive patients increases with the number of nodes collected

- Although there is no minimum number of nodes that should be recovered in an ALND specimen, 16 nodes should be regarded as a target to ensure a high level of confidence that the nodes are negative more difficult to find. ${ }^{22}$ The Edinburgh breast pathology service is consultant led, and all dissections were carried out by a consultant member of the breast pathology team (three consultants) or a specialist registrar under supervision. There is no trimming of breast specimens by biomedical scientists in Edinburgh at the present time or during the period of study. We do not have precise data on the relative split of dissections by consultants and specialist registrars, but would estimate approximately $70 \%$ to $30 \%$, respectively. Specialist registrars in our unit have a very clear understanding of the importance of node retrieval from ALNDs and are very closely supervised. Surgical factors must come into play in the node yield from these specimens, although the unit has a policy to perform level III dissections in every case. During the period of study, the Edinburgh Breast Unit was staffed by five consultant surgeons, one rotating specialist registrar, and a research fellow. We have no data on how the node count varied among this group of surgeons. The unit has an international reputation and adherence to surgical policy is very good. Those cases with very small numbers of nodes retrieved were probably low yielding because of surgical rather than pathological factors (for example, early cessation of the operation, undeclared previous node sampling, or undeclared previous neoadjuvant treatment), but we have no data to support these suppositions. The patients with known neoadjuvant chemotherapy have been excluded from the main study group of 520 patients.

"The more lymph nodes recovered the higher the proportion of node positive patients up to a plateau of between 16 and 20 nodes"

Our study shows a significant difference between the percentage of node positive patients with 16 or more and those with less than 16 lymph nodes collected. The more lymph nodes recovered the higher the proportion of node positive patients up to a plateau of between 16 and 20 nodes. More than 54\% of node positive patients in our study had 16 or more nodes collected. Although some centres set a desirable minimum for the numbers of nodes retrieved in an ALND specimen the evidence base for the said numbers is limited and has prompted our study. Our findings support a previous study on 236 patients with invasive breast cancer, which showed that the proportion of node positive patients increased with the number of nodes collected ${ }^{23}$; in contrast, a study of 499 axillary dissection specimens suggested that examining only the four to six largest lymph nodes was a reliable alternative staging method to identify patients in the pNO category. ${ }^{24}$ Our findings also support the findings of a larger study on 5125 patients with clinically negative nodes who underwent axillary dissection, which demonstrated that the number of lymph nodes collected, tumour grade, size, and site were all independent predictors of the risk of nodal involvement. A lower incidence of detected nodal involvement was evident for those patients with smaller tumours and fewer than 16 nodes examined. ${ }^{25}$ This supports our data in setting 16 to 20 lymph nodes as the target for recovery to place patients in the pNO staging category with confidence.

\section{ACKNOWLEDGEMENTS}

We are grateful to Mr G Wilson and Ms P Nimmo for assistance with data retrieval.

\section{Authors' affiliations}

J E A Somner, Edinburgh University Medical School, Edinburgh EH8 9AG, UK

J M J Dixon, Edinburgh Breast Unit, Western General Hospital, Edinburgh EH4 2XU, UK 
J S J Thomas, Department of Pathology, Western General Hospital, Edinburgh EH4 2XU, UK

\section{REFERENCES}

1 Bonadonna G, Hortobagyi GN, Gianni AM. Textbook of breast cancer, a clinical guide to therapy, 2nd ed. London: Martin Dunitz, 2001

2 Golshirsh A, Wood WC, Senn H-J, et al. Meeting highlights international consensus panel on the treatment of primary breast cancer. J Natl Caner Inst 1995:87:1441-5.

3 Henson De, Ries L, Freedman LS, et al. Relationship among outcome, stage of disease and histologic grade for 22,616 cases of breast cancer: the basis for a prognostic index. Cancer 1991;68:2142-9.

4 Fisher ER, Anderson S, Redmond C, et al. Pathologic findings from the National Surgical Adjuvant Breast Project Protocol B-06: 10-year pathologic and clinical prognostic discriminants. Cancer 1993;71:2507-14.

5 Veronesi U, Galimberti V, Zurrida S, et al. Prognostic significance of number and level of axillary nodal metastases in breast cancer. Breast 1993;2:224-8.

6 Jatoi I, Hilsenbeck SG, Clark GM, et al. Significance of axillary lymph node metastasis in primary breast cancer. J Clin Oncol 1999;17:2334-40.

7 Nishizaki T, DeBries S, Chew K, et al. Genetic alterations in primary breast cancers and their lymph node metastases. Direct comparison by modified comparative genomic hybridization. Genes Chromosomes Cancer 1997; 19:267-72.

8 Maunsell E, Brisson J, Deshenes I. Arm problems and psychological distress after surgery for breast cancer. Can J Surg 1993;36:315-20.

9 Singletary SE. Management of the axilla in early stage breast cancer. In: Perry MC, ed. American Society of Clinical Oncology educational book. Alexandria, VA: American Society of Clinical Oncology, 1998:132-41.

10 Carter CL, Allen C, Henson DE. Relation of tumour size, lymph node status, and survival in 24,740 breast cancer cases. Cancer 1989;63:181-7.

11 Fitzgibbons PL, Page DL, Weaver D, et al. Prognostic factors in breast cancer College of American Pathologists consensus statement 1999. Arch Pathol Lab Med 2000; 124:966-78.

12 Goldstein NS, Sanford W, Coffey M, et al. Lymph node recovery from colorectal resection specimens removed for adenocarcinoma. Trends over time and a recommendation for a minimum number of lymph nodes to be recovered. Am J Clin Pathol 1996;106:209-16.
13 Cianchi F, Palomba A, Boddi V, et al. Lymph node recovery from colorectal tumour specimens: recommendation for a minimum number of lymph nodes to be examined. World J Surg 2002;26:384-9

14 Goldstein NS. Lymph node recoveries from 2427 pT3 colorectal resection specimens spanning 45 years: recommendations for a minimum number of recovered lymph nodes based on predictive probabilities. Am J Surg Pathol 2002;26:179-89.

15 Pheby DFH, Levine DF, Pitcher RW, et al. Lymph node harvests directly influence the staging of colorectal cancer: evidence from a regional audit. J Clin Pathol 2004:57:43-7.

16 Ahlgren J, Holmberg L, Bergh J, et al. Five node biopsy of the axilla: an alternative to axillary dissection of levels I-II in operable breast cancer. Eur J Surg Oncol 2002;28:97-102.

17 Veronesi U, Paganelli G, Galimberti V, et al. Sentinel node biopsy to avoid axillary dissection in breast cancer with clinically negative lymph nodes. Lancet 1997;349:1861-7.

18 Weir L, Speers C, D'yachkova Y, et al. Prognostic significance of the number of axillary lymph nodes removed in patients with node-negative breast cancer. J Clin Oncol 2002;20:1793-9.

19 van der Wal BC, Butzelaar RM, van der Meij S, et al. Axillary lymph node ratio and total number of removed lymph nodes: predictors of survival in stage I and II breast cancer. Eur J Surg Oncol 2002;28:481-9.

20 Sosa JA, Diener-West M, Gusev Y, et al. Association between extent of axillary node clearance and survival in patients with stage I breast cancer. Ann Surg Oncol 1998;5:140-9.

21 Saha S, Farrar WB, Young DC, et al. Variation in axillary node dissection influences the degree of nodal involvement in breast cancer patients. J Surg Oncol 2000:73:134-7.

22 Baslaim MM, Al Malik OA, Al-Sobhi SS, et al. Decreased axillary lymph node retrieval in patients after neoadjuvant chemotherapy. Am J Surg 2002; 184:299-301

23 Kutiyanawala MA, Sayed M, Stotter A, et al. Staging the axilla in breast cancer: an audit of lymph-node retrieval in one U.K. regional centre. Eur J Surg Oncol 1998;24:280-2.

24 Cserni G. The reliability of sampling three to six nodes for staging breast cancer. J Clin Pathol 1999;52:681-3.

25 Voogd AC, Coebergh JW, Repelaer van Driel OJ, et al. The risk of nodal metastases in breast cancer patients with clinically negative lymph nodes: a population-based analysis. Breast Cancer Res Treat 2000;62:63-9. 